\title{
Combined application of Robicsek procedure and thermoreactive nitinol clips for treating a patient with sternal dehiscence
}

\author{
Habib Cakir*, Yüksel Besir, Ismail Yurekli, Koksal Donmez, Ersin Celik, Mert Kestelli, \\ Ufuk Yetkin, Ali Gurbuz \\ Department of Cardiovascular Surgery, Katip Celebi University Izmir Ataturk Training and Research Hospital, Izmir, Turkey \\ Email: habibcakir35@hotmail.com
}

Received 24 July 2013; revised 11 August 2013; accepted 19 August 2013

Copyright (C) 2013 Habib Cakir et al. This is an open access article distributed under the Creative Commons Attribution License, which permits unrestricted use, distribution, and reproduction in any medium, provided the original work is properly cited.

\begin{abstract}
Postopereative sternal dehiscence is one of the most important complications of median sternotomy which may cause pulmonary dysfunction and mediastinitis. We are reporting a patient with sternal dehiscence after coronary artery bypass surgery that underwent application of thermoreactive nitinol clips in addition to Robicsek procedure. Using thermoreactive nitinol clips method together with Robicsek procedure may be a valuable technique for preventing recurrent sternal dehiscence for patients who are candidates of noninfective recurrent sternal dehiscence.
\end{abstract}

Keywords: Sternal Dehiscence; Robicsek Procedure; Thermoreactive Nitinol Clips

\section{INTRODUCTION}

Median sternotomy is the most commonly performed procedure in cardiac surgery. Postopereative sternal dehiscence is one of the most important complications of median sternotomy which may cause pulmonary dysfunction and mediastinitis. Robicsek procedure or thermoreactive nitinol treatment may be preferred for treating non-infective sternal dehiscence [1]. There is not enough data about combined usage of these two techniques in the literature.

In this case report, we present a patient with non-infective sternal dehiscence after coronary artery bypass surgery that underwent application of thermoreactive nitinol clips in addition Robicsek procedure.

\section{CASE PRESENTATION}

A 72-year-old male patient was referred to cardiology

${ }^{*}$ Corresponding author. clinic with complaints of exertional angina. Coronary angiography revealed three vessel disease and coronary artery bypass surgery was planned. Mild mitral valve regurgitation was present at transthoracic echocardiography. His left ventricular ejection fraction was $65 \%$. He underwent coronary artery bypass surgery and three vessels were revascularized with saphenous vein graft (Left anterior descending artery, obtuse margin 1 and obtuse margin 2 arteries). Ventricular fibrillation occurred after closure of the sternum. After reopening sternum, cardiopulmonary bypass was reestablished under emergency circumstances. All vein grafts were patent and there was not any electrolyte imbalance. Inotropic support was administered and intraaortic balloon pump was inserted. Weaning from cardiopulmonary bypass was successful with support of intraaortic balloon pump. Sternum was not reapproximated due to myocardial edema. Patient was transported to intensive care unit. On first postoperative day, revision surgery and approximation of sternum were performed. Inotropic agents and intraaortic balloon pump support were terminated on fifth postoperative day. Patient was transported to cardiovascular surgery ward on postoperative sixth day. Due to fever, serous discharge from sternal incision and sternal dehiscence patient underwent revision operation on 14th postoperative day. Sternum's left side was broken into three pieces caused by sternal wires whipsaw effect. There were not any sign of deep or superficial mediastinal infection. Debris at anterior mediastinum were removed and cultures were taken. Robicsek sternoplasty was decided for left half of sternum. Right half was intact and no additional procedures were required. After sternoplasty, sternum was approximated conventionally with steel wires (Figure 1). After approximation minor defects on coaptation surfaces of sternum were noticed (Figure 1). Application of thermoreactive nitinol clips 
was decided. Three thermoreactive nitinol clips were applied to defected areas. Complete coaptation of sternum was achieved after procedure (Figure 2). Wound cultures and debris cultures were negative. He is still under follow-up of our outpatient clinic with no sternal dehiscence.

\section{DISCUSSION}

Sternal dehiscence is one of the most important complications of median sternotomy and it is incidence is 0.39\%. New York Heart Association Class IV, obesity and chronic obstructive pulmonary disease are considered to be the most important preoperative risk factors for sternal dehiscence [2]. None of these risk factors

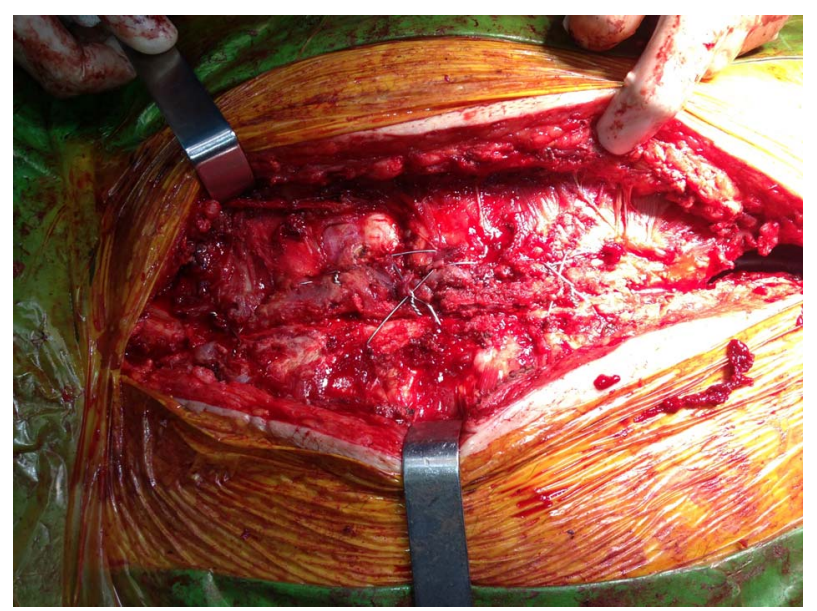

Figure 1. After approximation minor defects on coaptation surfaces of sternum.

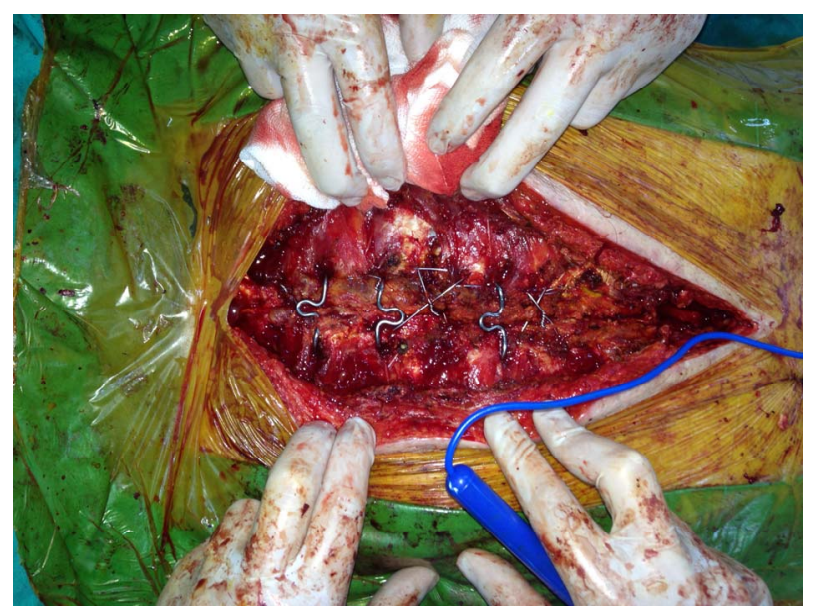

Figure 2. Complete coaptation of sternum after thermoreactive nitinol clips application. were present in our patient. Sternal dehiscence may cause complications like mediastinitis and pulmonary dysfunction. This causes necessity of early treatment as we have treated our patient.

Thermoreactive nitinol treatment is being widely used for treating non-infective sternal dehiscence instead of Robicsek procedure and succesful results are reported [1]. In one study, applying nitinol clips for approximation of median sternotomy had better clinical results than conventional methods [3]. There is not enough data about combined usage of these two techniques in the literature. We have stabilized left part of sternum with Robiscek procedure and sternum was wired conventionally. Although sternum was stable, there were defects at coaptation surfaces caused by fracture. For reducing the risk of recurrent sternal dehiscence, application of thermoreactive nitinol clips was decided intraoperatively. Three nitinol thermoreactive clips were applied to defected areas. After application, defects on coaptation surfaces of sternum were lost and greater stability of sternum was achieved.

In this case, we observed that thermoreactive nitinol clips application increased sternal stability by by Robiscek procedure. In conclusion, for patients with non-infective sternal dehiscence who are candidates of recurrence, combined treatment of Robiscek procedure and thermoreactive nitinol clips application may be the method of choice to reduce recurrent sternal dehiscence. However, to obtain more reliable evidence, prospective randomized controlled trials are needed.

\section{REFERENCES}

[1] Gucu, A., Toktas, F., Eris, C., Ata, Y. and Turk, T. (2012) Nitinol thermoreactive clips for secondary sternal closure in cases of noninfective sternal dehiscence. Texas Heart Institute Journal, 39, 513-516.

[2] Olbrecht, V.A., Barreiro, C.J., Bonde, P.N., Williams, J.A., Baumgartner, W.A., Gott, V.L., et al. (2006) Clinical outcomes of noninfectious sternal dehiscence after median sternotomy. The Annals of Thoracic Surgery, 82, 902-907. doi:10.1016/j.athoracsur.2006.04.058

[3] Bejko, J., Tarzia, V., De Franceschi, M., Bianco, R., Castoro, M., Bottio, T., et al. (2012) Nitinol flexigrip sternal closure system and chest wound infections: Insight from a comparative analysis of complications and costs. The Annals of Thoracic Surgery, 94, 1848-1853. doi:10.1016/j.athoracsur.2012.08.032 